\title{
PREVALÊNGIA DE CÁRIE EM ESCOLARES DA ZONA RURAL DE INDAIABIRA, MINAS GERAIS, BRASII
}

Caries prevalenee among students in the rural area of Indaiabira, Minas Gerais, Brazil

\author{
Maurício da Rocha Dourado ${ }^{1}$, João Henrique Aguiar Rebelo², \\ Aline Leal Rocha ${ }^{3}$, Thalita Thyrza de Almeida Santa-Rosa ${ }^{4}$
}

\begin{abstract}
RESUMO
O agravo que mais frequentemente afeta a saúde bucal da população é a cárie dentária, considerada um problema de saúde pública. Este trabalho objetivou conhecer a experiência de cárie entre escolares do município de Indaiabira-MG, visando subsidiar o planejamento de ações de promoção, prevenção e restauração da saúde bucal dessa população. Participaram os escolares residentes na zona rural, na faixa etária de 5 a 14 anos, sendo os dados colhidos nos prontuários odontológicos com autorização prévia da Secretaria Municipal de Saúde. Foram avaliados 637 escolares, 53,5\% eram do sexo masculino. Estavam livres de cárie 242 crianças, $38 \%$ do total avaliado. O índice ceo para a idade de 5 anos foi de 4,25 ( $\pm 3,219)$, e o CPOD para as idades de 12 e 14 anos foi respectivamente 4,19 $( \pm 2,721)$ e $4,86( \pm 2,92)$ com grande participação do componente cariado e necessidade de tratamento restaurador. É importante que se faça a intervenção nas idades iniciais, buscando prevenir a instalação da doença e de suas consequências na população adulta e idosa.
\end{abstract}

PALAVRAS-CHAVE: Levantamento Epidemiológico; Cárie Dentária; Escolares; Zona Rural.

\section{INTRODUÇÃO}

A Odontologia tem apresentado mudanças em suas ações, que passaram de medidas essencialmente curativas para uma abordagem mais abrangente, voltada para os determinantes do processo saúde-doença. Esse novo enfoque, por sua vez, tem repercutido na necessidade de

\begin{abstract}
The disorder that most often affects the oral health of the population is tooth decay, which is considered a public health problem. This study focused on dental caries experience among school children of Indaiabira, MG in order to support the planning of health promotion, prevention, and restoration of the oral health of this population. The participants were children, ages 5-14 years, living in the rural area. Data was gathered from the dental records with the prior permission of the Municipal Health Department. A total of 637 students were evaluated, $53.5 \%$ males, and 242 were caries-free, $38 \%$ of the total assessed. The DMF index for 5-yearolds was 4.25 ( \pm 3.219) and the DMFT at ages 12 and 14 years respectively was $4.19( \pm 2.721)$ and 4.86 ( \pm 2.92$)$, with a large proportion of decayed teeth and the need for restorative treatment. It is important to intervene at an early age to try to prevent the onset of this illness and its consequences in the adult and elderly populations.
\end{abstract}

KEYWORDS: Epidemiological Survey; Dental Caries; School Children; Rural Area.

serem instituídas estratégias de atenção odontológica mais precocemente, como aquelas junto às crianças de pouca idade - importantes para a redução das sequelas das doenças bucais mais prevalentes e do custo do tratamento das mesmas. ${ }^{1}$

No que diz respeito à saúde bucal, o agravo que mais frequentemente afeta a população é a cárie dentária, con-

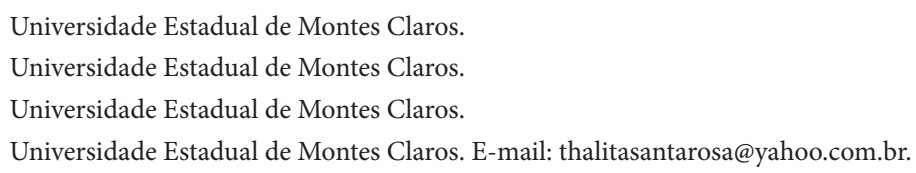


siderada um problema de saúde pública na maior parte do planeta, pois remete a quadros de infecção, dor, sofrimento e mutilação, diminuindo a qualidade de vida dos indivíduos. ${ }^{2}$

A cárie é uma doença multifatorial e crônica que ocorre quando há um desequilíbrio do processo dinâmico entre a estrutura dentária e o seu meio ambiente, sendo necessária a interação de três fatores como substrato cariogênico (dieta), microbiota bucal cariogênica e estrutura dentária suscetível para desencadear esse processo. Esta concepção da doença como multifatorial nos remete que para promover a saúde bucal das populações, é necessário levar em conta as variáveis biológicas, os fatores sociais e psicológicos, pois ter saúde não é somente uma escolha, mas uma condição determinada pelo meio social, cultural e econômico. ${ }^{3}$

Nos últimos anos, os padrões de cárie têm sido alterados nas diferentes regiões do mundo, principalmente entre crianças. $\mathrm{Na}$ maioria dos países desenvolvidos tem havido declínio na prevalência e severidade da doença, devido principalmente ao aumento da exposição ao flúor e a modificações no padrão e quantidade de consumo de açúcar, associados à melhoria nas condições de vida, maior acesso aos serviços odontológicos e ampliação das ações de promoção e educação em saúde bucal. O Brasil tem acompanhado essa tendência mundial, principalmente no que se refere à idade de 12 anos, quando comparados os principais levantamentos epidemiológicos realizados a nível nacional, entretanto, a cárie dentária ainda representa o principal problema de saúde bucal coletiva no país. $4,5,6,7,8,9$

O conhecimento do perfil de agravos à saúde, tradicional tarefa da saúde pública, é um indicador insubstituível para a provisão de serviços e de recursos, seja em termos de pessoal, de equipamentos, medicamentos e demais insumos usados em atividades de natureza preventiva, diagnóstica, terapêutica e de reabilitação. ${ }^{10}$

Apesar dos resultados animadores sobre o declínio da cárie, a maioria dos estudos foi realizada em capitais ou grandes cidades e, na revisão da literatura científica nacional, foram observados poucos dados epidemiológicos em saúde-doença bucal dos habitantes de pequenos municípios e sobre sua população rural. O conhecimento da distribuição da cárie nos municípios é de extrema relevância, pois esta representa um problema de saúde pública. Dentro deste contexto, conforme preconiza a lei 8080 de 1990, a epidemiologia deve ser utilizada nesse processo, a fim de que medidas apropriadas de controle das doenças sejam implementadas. ${ }^{11}$

Este trabalho objetivou conhecer a experiência de cárie entre escolares de 5 a 14 anos do município nortemineiro de Indaiabira, visando subsidiar o planejamento de ações de promoção, prevenção e restauração da saúde bucal dessa população.

\section{METODOLOGIA}

O município de Indaiabira está situado no norte de Minas Gerais e possui população de 7425 habitantes, $84,3 \%$ residentes na zona rural, e taxa de urbanização de 16,61\%. Possui ainda densidade demográfica de 7,8 habitantes $/ \mathrm{km}^{2}$, IDH de 0,571 , um dos menores do estado, e sistema de fluoretação das águas ausente na zona rural. ${ }^{12}$ No ano de 2010, entre os meses de agosto e novembro, foi realizado levantamento epidemiológico acerca da situação de saúde bucal dos escolares da zona rural deste município pelos cirurgiões-dentistas das três Equipes de Saúde da Família, ação prevista no Programa Saúde na Escola do governo federal em que o município foi contemplado. Durante o Estágio Supervisionado, disciplina obrigatória inserida no último período da graduação do Curso de Odontologia da Universidade Estadual de Montes Claros - Unimontes, com autorização prévia, os acadêmicos estagiários analisaram os dados levantados.

Trata-se de um estudo quantitativo e transversal, com a utilização de dados secundários, colhidos nas fichas clínicas do consultório odontológico. Para tanto, foram selecionados todos os alunos de 5 a 14 anos de idade, matriculados na rede pública municipal e residentes na zona rural, tendo como critério de exclusão as fichas incompletas, os que não se encontravam na faixa etária estudada e os que não residiam na zona rural. Obteve-se como critério de procedência (zona rural), o local de residência, independente da localização da escola. As variáveis foram construídas a fim de se obter CPO-D e ceo-d, necessidade de tratamento odontológico (selante, restauração ou extração por elemento dentário), condição dos primeiros molares permanentes e uso do serviço público odontológico, de acordo à idade e sexo.

A coleta de dados se deu no ambiente escolar por dois examinadores, de acordo com a área de abrangência de sua Equipe de Saúde da Família. O exame clínico epidemiológico se deu sob luz natural, com o auxílio de abaixadores de língua, estando os examinados sentados à frente do examinador, devidamente paramentado com equipamento de proteção individual. Os índices usados para a cárie dentária foram o ceo-d para dentição decídua e o CPO-D para dentição permanente, os quais consistem na soma dos dentes cariados, perdidos (ou dentes com extração indicada, quando decíduos) e obturados. Para cada elemento dental, foi indicado o tratamento quando necessário. Os critérios diagnósticos foram aqueles sugeridos pelo Informe Técnico número 20 da Secretaria Estadual 
de Saúde de Minas Gerais. O dente foi considerado hígido quando não havia nenhuma alteração visível, fluorose moderada ou severa, todas as lesões questionáveis, hipoplasia de esmalte, erosão e abrasão. Foram classificados como cariados apenas quando havia certeza da presença de cárie e quando cavitado. E os dentes com extração indicada, aqueles com cavidade ampla com suposta perfuração do assoalho, problemas múltiplos, e remanescentes radiculares.

\section{RESULTADOS}

Foram avaliados 637 escolares de 5 a 14 anos no município de Indaiabira. Do total, $53,5 \%$ eram do sexo masculino e 46, 5\% feminino. Apesar da amostra bem distribuída, a idade com maior número de participantes foi a de 10 anos (13,5\%). Estavam livres de cárie 242 crianças, 38\%
Para necessidade de tratamento restaurador foram selecionados os dentes cariados (passíveis de recuperação) e obturados com cárie, não diferenciando decíduos e permanentes. Os selantes foram indicados para dentes permanentes recém-erupcionados em pacientes com alta atividade de cárie.

Os dados foram analisados no programa estatístico SPSS versão para Windows 17.0®.

do total avaliado. Na tabela 1, pode ser observado o ataque de cárie nos escolares por idade e condição, por dente e por indivíduo. Nota-se que o valor total por indivíduo foi omitido uma vez que um mesmo indivíduo pode apresentar várias condições, superestimando o total, o que não acontece na análise por dente, já que cada dente aceita uma condição apenas.

Tabela 1 - Ataque pela cárie nos escolares da zona rural de Indaiabira-MG no período de agosto a novembro de 2010.

\begin{tabular}{|c|c|c|c|c|c|c|c|c|c|c|}
\hline & & \multicolumn{2}{|c|}{ CARIADOS } & \multicolumn{2}{|c|}{ PERDIDOS } & \multicolumn{2}{|c|}{$\begin{array}{l}\text { EXTRAÇÃO } \\
\text { INDICADA }\end{array}$} & \multicolumn{2}{|c|}{ OBTURADOS } & \multirow{2}{*}{$\begin{array}{r}\text { TOTAL } \\
\text { dente }\end{array}$} \\
\hline & & dente & indivíduo & dente & indivíduo & dente & indivíduo & dente & indivíduo & \\
\hline \multirow{2}{*}{$\begin{array}{c}5 \text { anos } \\
40 \\
(6,3 \%)\end{array}$} & $\mathrm{n}$ & 155 & 35 & 0 & 0 & 31 & 11 & 0 & 0 & 186 \\
\hline & $\%$ & 83,4 & 87,5 & 0 & 0 & 16,6 & 27,5 & 0 & 0 & 100 \\
\hline \multirow{2}{*}{$\begin{array}{c}6 \text { anos } \\
51 \\
(8 \%)\end{array}$} & $\mathrm{n}$ & 84 & 22 & 0 & 0 & 11 & 5 & 0 & 0 & 95 \\
\hline & $\%$ & 88,5 & 43,1 & 0 & 0 & 11,5 & 9,8 & 0 & 0 & 100 \\
\hline \multirow{2}{*}{$\begin{array}{c}7 \text { anos } \\
74 \\
(11,6 \%)\end{array}$} & $\mathrm{n}$ & 171 & 42 & 0 & 0 & 74 & 28 & 1 & 1 & 246 \\
\hline & $\%$ & 69,5 & 56,75 & 0 & 0 & 30 & 37,83 & 0,49 & 1,35 & 100 \\
\hline \multirow{2}{*}{$\begin{array}{c}8 \text { anos } \\
64 \\
(10 \%)\end{array}$} & $\mathrm{n}$ & 162 & 43 & 1 & 1 & 66 & 17 & 2 & 2 & 231 \\
\hline & $\%$ & 70,1 & 67,19 & 0,43 & 1,6 & 28,57 & 26,6 & 0,86 & 3,13 & 100 \\
\hline \multirow{2}{*}{$\begin{array}{c}9 \text { anos } \\
78 \\
(12,2 \%)\end{array}$} & $\mathrm{n}$ & 220 & 54 & 2 & 2 & 80 & 25 & 11 & 4 & 313 \\
\hline & $\%$ & 70,2 & 69,23 & 0,63 & 2,56 & 2,55 & 32,1 & 3,51 & 5,1 & 100 \\
\hline \multirow{2}{*}{$\begin{array}{c}10 \text { anos } \\
86 \\
(13,5 \%)\end{array}$} & $\mathrm{n}$ & 227 & 64 & 17 & 13 & 57 & 30 & 18 & 8 & 319 \\
\hline & $\%$ & 71,1 & 74,4 & 5,32 & 15,11 & 17,86 & 34,9 & 5,64 & 9,3 & 100 \\
\hline \multirow{2}{*}{$\begin{array}{c}11 \text { anos } \\
78 \\
(12,2 \%)\end{array}$} & $\mathrm{n}$ & 280 & 70 & 17 & 13 & 47 & 24 & 21 & 8 & 365 \\
\hline & $\%$ & 76,7 & 89,7 & 4,65 & 16,7 & 12,87 & 30,77 & 5,75 & 10,25 & 100 \\
\hline
\end{tabular}




\begin{tabular}{|c|c|c|c|c|c|c|c|c|c|c|}
\hline & & \multicolumn{2}{|c|}{ CARIADOS } & \multicolumn{2}{|c|}{ PERDIDOS } & \multicolumn{2}{|c|}{$\begin{array}{l}\text { EXTRAÇÃO } \\
\text { INDICADA }\end{array}$} & \multicolumn{2}{|c|}{ OBTURADOS } & \multirow{2}{*}{$\begin{array}{r}\text { TOTAL } \\
\text { dente }\end{array}$} \\
\hline & & dente & indivíduo & dente & indivíduo & dente & indivíduo & dente & indivíduo & \\
\hline \multirow{2}{*}{$\begin{array}{c}12 \text { anos } \\
62 \\
(9,7 \%)\end{array}$} & $\mathbf{n}$ & 227 & 55 & 13 & 10 & 19 & 13 & 27 & 13 & 286 \\
\hline & $\%$ & 79,3 & 88,7 & 4,54 & 16,12 & 6,64 & 20,96 & 9,44 & 20,96 & 100 \\
\hline \multirow{2}{*}{$\begin{array}{c}13 \text { anos } \\
62 \\
(9,7 \%)\end{array}$} & $\mathrm{n}$ & 199 & 45 & 24 & 13 & 12 & 6 & 42 & 18 & 277 \\
\hline & $\%$ & 71,8 & 72,58 & 8,66 & 20,96 & 4,33 & 9,67 & 15,1 & 29,1 & 100 \\
\hline \multirow{2}{*}{$\begin{array}{c}14 \text { anos } \\
42 \\
(6,6 \%)\end{array}$} & $\mathrm{n}$ & 158 & 38 & 18 & 12 & 13 & 6 & 28 & 13 & 217 \\
\hline & $\%$ & 72,8 & 90,47 & 8,29 & 28,57 & 6 & 14,28 & 6 & 30,9 & 100 \\
\hline TOTAL & & 1883 & 469 & 92 & 64 & 410 & 165 & 150 & 67 & 2535 \\
\hline
\end{tabular}

Fonte: Secretaria Municipal de Saúde de Indaiabira-MG.

O índice ceo-d para a idade de 5 anos foi de 4,25 $( \pm 3,219)$, e o CPOD para as idades de 12 e 14 anos foi respectivamente $4,19( \pm 2,721)$ e 4,86 ( $\pm 2,927)$. Não houve diferenças estatisticamente significantes entre os sexos.

O gráfico 1 revela a relação existente entre os dentes hígidos, cariados, perdidos e obturados, nas idades de 5 , 12 e 14 anos, considerando as extremidades analisadas ( 5 e 14 anos) e as idades índice de 5 e 12 anos. ${ }^{13}$ É importante salientar que o número de dentes decíduos é menor do que os da arcada permanente, o que pode superestimar o aumento de elementos hígidos quando comparadas as idades de 5 e 12 anos. A média e desvio padrão de dentes hígidos para essas idades foram respectivamente de 15,35 $( \pm 3,36)$ para 5 anos, $23,42( \pm 2,80)$ aos 12 anos e de 22,9 $( \pm 3,00)$ aos 14 anos.

Gráfico 1 - Valor absoluto de dentes cariados, perdidos, obturados e hígidos nas idades de 5, 12 e 14 anos, em escolares da zona rural do município de Indaiabira-MG entre os meses de agosto e novembro de 2010.

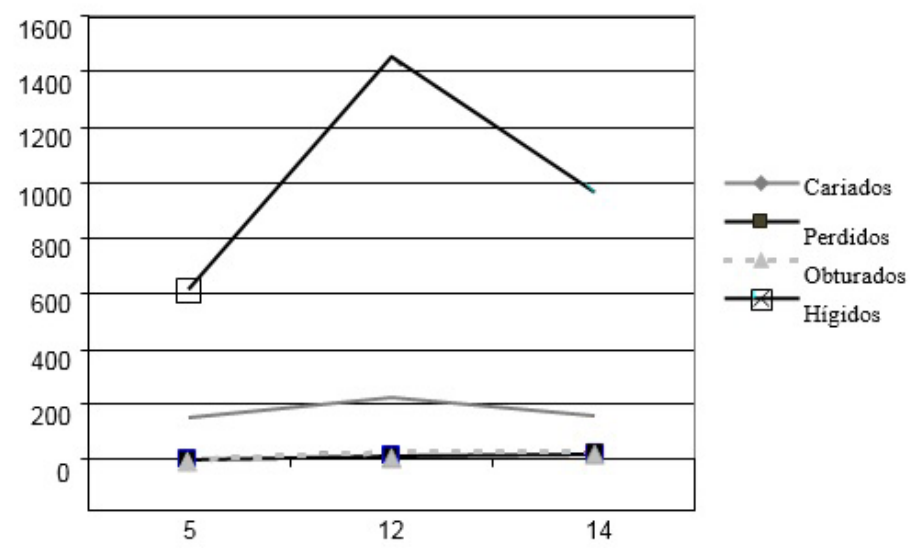

Fonte: Secretaria Municipal de Saúde de Indaiabira-MG. 
Quando analisado o uso do serviço odontológico, $40,8 \%$ dos escolares nunca haviam sido atendidos por um cirurgião-dentista. O gráfico 2 mostra o perfil de necessi- dade de tratamento dos escolares avaliados, ressaltando a grande necessidade de tratamento restaurador.

Gráfico 2 - Necessidade de Tratamento odontológico entre escolares da zona rural de Indaiabira-MG no período de agosto a novembro de 2010 .

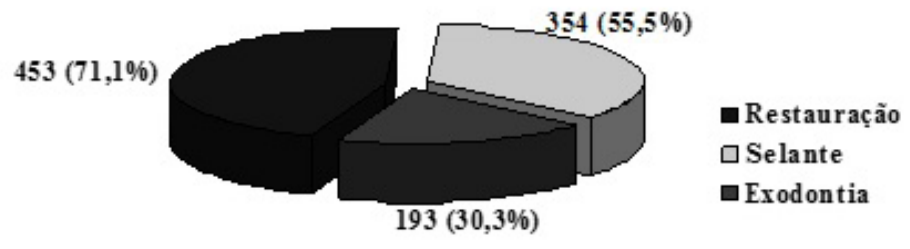

Fonte: Secretaria Municipal de Saúde de Indaiabira-MG.

A amostra também foi analisada quanto à condição do primeiro molar permanente, devido à sua importância na estabilidade oclusal de toda arcada. A variável foi utilizada para todas as idades, com as possibilidades de resposta hígido, cariado, perdido, obturado e não se aplica, para aqueles que ainda não apresentavam o dente permanente erupcionado. A tabela 2 explora estes dados por dente e por condição.

Tabela 2 - Condição do primeiro molar permanente em escolares da zona rural de Indaiabira-MG entre os meses de agosto e novembro de 2010 .

\begin{tabular}{|c|c|c|c|c|c|}
\hline & & $\begin{array}{c}\text { Primeiro Molar } \\
\text { Permanente Superior } \\
\text { Direito (16) }\end{array}$ & $\begin{array}{l}\text { Primeiro Molar } \\
\text { Permanente Superior } \\
\text { Esquerdo (26) }\end{array}$ & $\begin{array}{l}\text { Primeiro Molar } \\
\text { Permanente Inferior } \\
\text { Esquerdo (36) }\end{array}$ & $\begin{array}{c}\text { Primeiro Molar } \\
\text { Permanente Inferior } \\
\text { Direito (46) }\end{array}$ \\
\hline \multirow{2}{*}{ Hígido } & $\mathrm{n}$ & 338 & 329 & 241 & 245 \\
\hline & $\%$ & 59,2 & 57,7 & 41,6 & 42,6 \\
\hline \multirow{2}{*}{ Cariado } & $\mathrm{n}$ & 215 & 224 & 283 & 282 \\
\hline & $\%$ & 37,65 & 39,3 & 48,9 & 49,04 \\
\hline \multirow{2}{*}{ Perdido } & $\mathrm{n}$ & 8 & 11 & 34 & 28 \\
\hline & $\%$ & 1,4 & 1,9 & 5,87 & 4,86 \\
\hline \multirow{2}{*}{ Obturado } & $\mathrm{n}$ & 10 & 6 & 21 & 20 \\
\hline & $\%$ & 1,75 & 1,1 & 3,62 & 3,5 \\
\hline
\end{tabular}

Fonte: Secretaria Municipal de Indaiabira-MG. 


\section{DISCUSSÃO}

A cárie dentária é uma doença crônica que traz desconforto e influi na qualidade de vida das pessoas, e ainda necessita de atenção especial pela sua alta prevalência e severidade, principalmente em grupos populacionais específicos, como crianças da zona rural. ${ }^{14}$ É uma doença multifatorial que está apresentando tendência mundial de declínio, sobretudo em países desenvolvidos. Por outro lado, em países subdesenvolvidos, a baixa condição socioeconômica da população pode ser um agravante direto para o aumento da prevalência da doença cárie. ${ }^{15}$ Somando-se a isso, a dificuldade de acesso a dentifrícios e água fluoretada, a população se torna ainda mais susceptível à instalação dessa doença.

Os benefícios do desenvolvimento, tanto no Brasil como em outros países, são distribuídos de modo desigual à população, atingindo em geral, as áreas urbanas antes que as rurais, onde é maior a dificuldade em atender as necessidades de saúde da população. ${ }^{14}$

Neste estudo, 38\% da amostra estava livre de cárie, dado superior ao encontrado por Mereilles et al, ${ }^{11} \mathrm{em}$ escolares de 6 e 12 anos, no município de Cordeirópolis-SP, por Rigo et al, ${ }^{5}$ em escolares de 12 anos, em municípios do norte do Rio Grande do Sul, com e sem fluoretação das águas, superior ao valor encontrado por Menezes et $\mathrm{al},{ }^{16}$ na zona rural de Caruaru-PE e ainda por Abreu et al ${ }^{9}$ em escolares do meio rural de Itaúna-MG. Este dado foi menor do que o encontrado por Amaral et al, ${ }^{17} \mathrm{em}$ seus estudos com escolares do município de Rafard-SP. A redução dos índices de cárie no Brasil foi simultânea a uma crescente desigualdade na distribuição da doença, com níveis mais elevados, afetando as áreas mais submetidas à privação socioeconômica, caracterizando a polarização da doença que consiste na concentração da maior parte da cárie ou das necessidades de tratamento odontológico em uma pequena parcela da população. ${ }^{16}$

Quanto à distribuição de ataque pela cárie, assim como em outros estudos, ${ }^{18}$ os componentes com maior participação no CPOD/CEO foram os cariados e extração indicada, ou seja, tratamentos a realizar, enquanto os tratamentos já realizados, como perdidos e obturados tiveram menor participação. Isso revela a grande necessidade de assistência curativa pela população. Essa relação também foi encontrada por Tuon et al, ${ }^{14}$ em escolares de 7 a 14 anos da zona rural do município de Jacinto Machado-SC, por Amaral et al, ${ }^{17}$ nas crianças de 5 anos em Rafard-SP e por Menezes et al, ${ }^{16}$ na zona rural de Caruaru-PE. Esses resultados demonstram a necessidade de programas com atendimento coletivo preventivo e de educação para a saúde bucal, que sejam mais eficientes, para se reduzir o índice de ataque da doença na população jovem.

Em relação aos componentes do índice, observa-se uma regularidade no número de dentes cariados com o avançar da idade, e os obturados e perdidos apresentam um leve aumento com a idade. Diferente do observado por Freire et al, ${ }^{4}$ em escolares de Goiânia-GO, onde havia um predomínio de dentes cariados dos 6 aos 8 anos e de dentes obturados, a partir dos 9 anos. Porém, assim como neste estudo, a prevalência de dentes perdidos aumentou com a idade, representando porção significativa do CPOD.

O ceo-d encontrado para a idade de 5 anos foi de $4,25( \pm 3,219)$, valor maior do que encontrado por Menezes et al, ${ }^{16}$ em Caruaru-PE (3,0), por Amaral et al, ${ }^{17} \mathrm{em}$ Rafard-SP $(2,45 \pm 3,45)$, por Abreu et al, ${ }^{9} \mathrm{em}$ escolares do meio rural de Itaúna $(4,0 \pm 3,46)$, por Tuon et al, ${ }^{14}$ na zona rural de Jacinto Machado-SC $(3,5 \pm 3,7)$ e por Ruiz et al, ${ }^{19}$ em Leme-SP $(1,99)$.

Aproximadamente $70 \%$ dos países no mundo alcançaram a meta de apresentar três dentes ou menos com experiência de cárie aos 12 anos de idade, proposta pela Organização Mundial de Saúde (OMS) há 20 anos. ${ }^{20} \mathrm{O}$ município de Indaiabira ainda não conseguiu atingir essa meta, uma vez que o CPOD para esta idade foi de $4,19( \pm 2,721)$. Este valor foi maior do que o encontrado por Sampaio et al, ${ }^{21} \mathrm{em}$ uma área rural da Paraíba, onde existia concentração natural de flúor $(2,5)$, por Amaral et al, ${ }^{17}$ em Rafard-SP $(2,47 \pm 2,52)$, por Regis-Aranha et al, ${ }^{22} \mathrm{em}$ Boa Vista-RO $(3,2 \pm 2,9)$, por Ruiz et al, ${ }^{19} \mathrm{em}$ LemeSP $(1,97)$, por Alves Sá e Vasconcelos, ${ }^{3}$ no município de Verdejante-PE (3,2), por Moura et al, ${ }^{7}$ em Campina Grande-PB (2,09), por Traebert et al, ${ }^{23}$ em Blumenau-SC $(1,46)$, por Gushi et al, ${ }^{8}$ no estado de São Paulo $(2,52)$ e por Traebert et al, ${ }^{24}$ nos municípios rurais de São João do Sul $(1,91)$ e Treviso $(1,84)$. O CPOD em Indaiabira foi menor do que o encontrado por Freysleben et al, ${ }^{6}$ quando analisando a região Sul do Brasil $(6,25)$ e por Freire et al, ${ }^{4}$ na zona urbana de Goiânia-GO $(4,59)$.

Aos 14 anos, o CPOD foi de 4,86 ( $\pm 2,927)$, maior que o encontrado por Menezes et al, ${ }^{16}$ no município de Caruaru-PE $(1,67)$, por Tuon et al, ${ }^{14}$ na zona rural de Jacinto Machado-SC $(4,8 \pm 3,8)$, por Moreira et al, ${ }^{18}$ na cidade de Paulínia-SP $(4,3)$. Este dado foi menor que o CPOD encontrado por Abreu et al, ${ }^{9}$ na zona rural de Itaúna-MG $(6,94 \pm 1,55)$.

Estes dados discrepantes da literatura citada demonstra a gravidade da instalação da doença cárie e pode refletir a ausência de água fluoretada na zona rural deste município e a dificuldade de acesso ao flúor em qualquer forma que seja, à educação em saúde, a programas preventivos e ao serviço público assistencial. Do total de escolares ava- 
liados, 40,8\% nunca haviam sido atendidos por um cirurgião-dentista. Existe a necessidade de os profissionais da Odontologia assumirem uma postura de responsabilidade social em relação a uma população que não utiliza ou utiliza de forma irregular as suas ações. Profundas mudanças sociopolíticas, conceituais, pedagógicas e práticas tornamse necessárias para a Odontologia brasileira, sendo exigidos novos papéis profissionais do cirurgião-dentista, frente aos processos de geração das desigualdades sociais em saúde bucal. ${ }^{1}$

Dentre as necessidades de tratamento, os escolares da zona rural do município de Indaiabira-MG apresentaram maior necessidade de restaurações seguidas por selante. Foi detectado um grande número de dentes cariados e uma parcela significante da população $(40,8 \%)$ sem atendimento odontológico. A grande necessidade de selante pode ser resultado de uma grande atividade de cárie, sendo um mecanismo dificultador para o estabelecimento da doença nessas crianças. Já no estudo conduzido por Meirelles et al, ${ }^{11}$ em Cordeirópolis-SP, a principal necessidade foi de selantes oclusais, seguidos de tratamento restaurador. Em seu estudo com escolares de 6 e 12 anos, em Blumenau-SC, Traebert et al ${ }^{23}$ encontraram uma maior necessidade de tratamento restaurador, assim como nessa população.

Os primeiros molares permanentes são os principais dentes das arcadas dentárias por serem considerados a "chave da oclusão". Devido à sua morfologia, principalmente na superfície oclusal, são os dentes mais susceptíveis à cárie dentária e, por conseguinte, são os primeiros dentes a serem extraídos. É, portanto, necessário empregar os mais variados métodos de prevenção, evitando-se a perda e, geralmente, perda precoce desses dentes. ${ }^{18} \mathrm{Em}$ relação à localização do dente no arco dentário, a prevalência de cárie dentária foi maior nos inferiores, fato explicado na literatura pela facilidade maior de estagnação de placa nos inferiores.

Neste estudo, seguindo a tendência dos outros dentes, o CPOD para primeiros molares permanentes teve maior participação o componente cariado, diferente do encontrado por Moreira et al, ${ }^{18} \mathrm{em}$ seu estudo com escolares do interior do estado de São Paulo, onde prevaleceu o componente restaurado.

Considere-se que a população incluída no presente estudo foi constituída de escolares de escolas públicas, possivelmente representando um grupo de baixa condição socioeconômica. $\mathrm{O}$ estudo da relação entre indicadores de saúde e níveis socioeconômicos tem mostrado, em geral, uma relação diretamente proporcional, isso é, quanto pior o nível socioeconômico, mais comum em áreas rurais, piores os indicadores de saúde. Este postulado também se aplica para os indicadores de saúde bucal, especialmente para a cárie dentária. ${ }^{14}$

A avaliação das condições de saúde bucal da população, em geral, é importante por possibilitar o planejamento de ações socialmente adequadas e direcionar os recursos para grupos com níveis mais elevados de necessidades, contemplando princípios norteadores das políticas públicas de saúde atuais, como a equidade e a resolutividade. ${ }^{1}$ É importante salientar que alguns pontos como a falta de calibração dos examinadores e a coleta de dados em uma ficha clínica não específica para um levantamento epidemiológico podem ter provocado vieses nos resultados, superestimando ou subestimando-os. Porém os resultados apresentados demonstram a necessidade de maior intervenção na saúde bucal desses escolares, seja esta como prevenção, promoção ou reabilitação.

\section{CONCLUSÃO}

A população de Indaiabira-MG apresentou alto índice de cárie e consequentemente altos índices ceo-d e CPOD para as idades avaliadas. Os dados mostram que não há queda na porção de dentes cariados com o avançar da idade, seguido de um pequeno aumento dos elementos perdidos e obturados. Isso demonstra a falta de acesso ao serviço e às políticas públicas preventivas. É importante que se faça a intervenção nas idades iniciais, buscando prevenir a instalação da doença e de suas consequências na população adulta e idosa.

\section{Agradecimentos}

À FAPEMIG pelo apoio financeiro.

\section{REFERÊNCIAS}

1. Massoni ACLT, Vasconcelos FMN, Katz CRT, Rosenblatt A. Use of dental services and treatment needs of 5-to-12-year-old children in the city of Recife. Pernambuco, Brazil. Rev Odontol UNESP. 2009; 38(2):73-8.

2. Narvai PC. Cárie dentária e flúor: uma relação do século XX. Rio de Janeiro. Ciência \& Saúde Coletiva. 2000; 5(2):381-392.

3. Alves de Sá LC, Vasconcelos MMVB. Levantamento das necessidades odontológicas em crianças de 12 anos da zona urbana do município de Verdejante-PE. Odontologia. Recife. Clín.-Científ. 2008 out./dez.; 7(4):325-329.

4. Freire MCM. Prevalência de cárie e necessidades de tra- 
tamento em escolares de seis a doze anos de idade. Goiânia, GO, Brasil: 1994. Rev. Saúde Pública. 1997; 31(1):4452.

5. Rigo L, Abegg C, Bassani DG. Cárie dentária em escolares residentes em municípios do Rio Grande do Sul, Brasil, com e sem fluoretação nas águas. Rev Sul-Bras Odontol. 2009 mar.; 7(1):57-65.

6. Freysleben GR, Peres MAA, Marcenes W. Prevalência de cárie e CPO-D médio em escolares de doze a treze anos de idade nos anos de 1971 e 1997, região Sul, Brasil. Rev. Saúde Pública. 2000; 34(3):304-08.

7. Moura C, Cavalcanti AL, Bezerra PKM. Prevalência de cárie dentária em escolares de 12 anos de idade, Campina Grande, Paraíba, Brasil: enfoque socioeconômico. Rev. Odonto Ciênc. 2008; 23(3):256-262.

8. Gushi LL, Rihs LB, Soares MC, Forni TIB, Vieira V, Wada RS, et al. Cárie dentária e necessidades de tratamento em adolescentes do estado de São Paulo. 1998 e 2002. Rev Saúde Pública. 2008; 42(3):480-6.

9. Abreu de MHNG, Pordeus IA, Modena CM. Cárie dentária entre escolares do meio rural de Itaúna (MG), Brasil. Rev Panam Salud Publica. 2004; 16(5):334-44.

10. Martins AMEBL, Melo FS, Fernandes FM, Boa Sorte JA, Coimbra LGA, Batista RC. Levantamentos epidemiológicos brasileiros das condições de saúde bucal. Rev Unimontes Científica Montes Claros. 2005 jan./jun.; 7(1).

11. Meirelles MPM, Hoffmann RHS, de Souza MLR. Prevalência de cárie e necessidades de tratamento em escolares de Cordeirópolis, SP, Brasil. Revista de Odontologia da Universidade Cidade de São Paulo. 2007 set./dez.; 19(3):246-9.

12. Instituto Brasileiro de Geografia e Estatística. Brasil. [Citado 2010 dez.]. Disponível em: < http://www.ibge. gov.br/home/default.php>.

13. Brasil, Ministério da Saúde - Coordenação Nacional de Saúde Bucal. Condições de Saúde Bucal Da População Brasileira - Projeto SB Brasil 2003 - Resultados principais. [Citado 2010 dez.]. Disponível em: <HTTP:/ / portal,saude.gov.br/saude/arquivos/pdf/relatorio_brasil_sorridente.pdf $>$.

14. Tuon ACLF, Lacerda JT, Traebert JL. Prevalência de cárie em escolares da zona rural de Jacinto Machado, SC, Brasil. Pesquisa Brasileira em Odontopediatria e Clínica Integrada. 2007; 7(1):279-86.

15. Cortelli SC, Cortelli JR, Prado JS, Aquino DR, Jorge AOC. Fatores de risco a cárie e CPOD em crianças com idade escolar. Brasil. Ciênc Odontol. 2004 abr./jun.; 7(2):75-82.

16. Menezes VA, Maciel MAS, Santos VS, Leite AF, Martins VM, Granville-Garcia AF. Cárie dental e hábitos alimentares em escolares de uma zona rural de Caruaru-PE. Odonto. 2010; 18(36):83-94.

17. Amaral RC, Hofmann RHC, Cypriano S, Sousa MLR, Silva AAZ. Prevalência de cárie e necessidades de tratamento em pré-escolares e escolares de Rafard - SP - Brasil. Ciênc Odontol Bras. 2006 abr./jun.; 9(3):87-93.

18. Moreira BW, Pereira AC, Oliveira SP. Avaliação da prevalência de cárie dentária em escolares de localidade urbana da região Sudeste do Brasil. Rev. Saúde Pública. 1996; 30(3):280-4.

19. Ruiz LA, Rihs LB, Sousa MLR, Hildebrand L, Felizatti RC. Declínio da cárie dentária em escolares entre 1998 e 2004 em Leme, São Paulo, Brasil. RGO. 2009; 57(2):14150.

20. Nishi M, Stjernswärd J, Carlsson P, Bratthall D. Caries experience of some countries and areas expressed by Significant Caries Index. Community Dent Oral Epidemiol. 2002; 30(4):296-301.

21. Sampaio FC, Nazmul Hossain ANM, Von De Feher FR, Arneber GP. Dental caries and sugar intake of children from rural areas with different water fluoride levels in Paraíba, Brazil. Community Dent Oral Epidemiol. 2001; 28(4):307-13.

22. Régis-Aranha LA, Rebelo MAB, de Souza SMFM, Parente RCP. Cárie dentária em escolares de 12 anos de idade de Boa Vista, Roraima, Brasil. Cad. Saúde Pública, Rio de Janeiro. 2008 out.; 24(10):2449-2450.

23. Traebert J, Peres MA, Marcenes W, Galesso E, Zabot NE. Prevalência e severidade da cárie dentária. Revista de Saúde Pública. 2001; 35:283-288.

24. Traebert JL, Suarez CS, Onofri DA, Marcenes WS. Prevalência e severidade de cárie dentária e necessidade de 
tratamento odontológico em pequenos municípios brasileiros. Cad Saúde Pública. 2002; 18(3):817-21.

25. Moreira PV, Rosenblatt A, Passos IA. Prevalência de cárie dentária em adolescentes de escolas públicas e privadas da cidade de João Pessoa, Paraíba, Brasil. Ciênc Saúde Colet. 2007; 12:1229-36.

26. World Health Organization. Dental caries levels at 12 years. Geneve; 1994.

Submissão: julho de 2014

Aprovação: julho de 2016 